\title{
Safety and Efficacy of Extremely Low LDL-Cholesterol Levels and Its Prospects in Hyperlipidemia Management
}

\author{
Dhrubajyoti Bandyopadhyay (DD, ${ }^{1}$ Arshna Qureshi, ${ }^{2}$ Sudeshna Ghosh, ${ }^{3}$ Kumar Ashish, \\ Lyndsey R. Heise, ${ }^{5}$ Adrija Hajra, ${ }^{6}$ and Raktim K. Ghosh ${ }^{7}$ \\ ${ }^{1}$ Department of Internal Medicine, Mount Sinai St Luke's Roosevelt, New York, NY, USA \\ ${ }^{2}$ Department of Medicine, Lady Hardinge Medical College, New Delhi, India \\ ${ }^{3} I P G M E R$, Kolkata, India \\ ${ }^{4}$ The University of Texas MD Anderson Cancer Center, Houston, TX, USA \\ ${ }^{5}$ Department of Internal Medicine, University of Nebraska Medical Center, Omaha, NE, USA \\ ${ }^{6}$ Department of Internal Medicine, IPGMER, Kolkata, India \\ ${ }^{7}$ Division of Cardiovascular Diseases, Metrohealth Medical Center, Case Western Reserve University, Cleveland, OH, USA \\ Correspondence should be addressed to Dhrubajyoti Bandyopadhyay; drdhrubajyoti87@gmail.com
}

Received 19 November 2017; Accepted 14 March 2018; Published 23 April 2018

Academic Editor: Gerhard M. Kostner

Copyright (c) 2018 Dhrubajyoti Bandyopadhyay et al. This is an open access article distributed under the Creative Commons Attribution License, which permits unrestricted use, distribution, and reproduction in any medium, provided the original work is properly cited.

\begin{abstract}
The risk of cardiovascular disease has been reported to have a linear relationship with LDL levels. Additionally, the currently recommended LDL target goal of $70 \mathrm{mg} / \mathrm{dl}$ does not diminish the CV risk entirely leaving behind some residual risk. Previous attempts to maximally lower the LDL levels with statin monotherapy have met dejection due to the increased side effects associated with the treatment. Nevertheless, with the new advancements in clinical medicine, it has now become possible to bring down the LDL levels to as low as $15 \mathrm{mg} / \mathrm{dl}$ using PCSK9 monoclonal antibodies alone or in combination with statins. The development of inclisiran, siRNA silencer targeting PCSK9 gene, is a one step forward in these endeavors. Moreover, various studies aiming to lower the CV risk and mortality by lowering LDL levels have demonstrated encouraging results. The current challenge is to explore this arena to redefine the target LDL levels, if required, to avoid any suboptimal treatment. After thorough literature search in the PubMed, Embase, Scopus, and Google Scholar, we present this article to provide a brief overview of the safety and efficacy of lowering LDL below the current goal.
\end{abstract}

\section{Introduction}

Hyperlipidemia has always been a topic of interest owing to the concomitant increased risk of adverse cardiovascular events. Coronary artery disease, a leading cause of death in the United States with almost 400,000 deaths/year, is found to be strongly associated with hyperlipidemia [1]. Moreover, increased LDL levels are found to be positively correlated with the increased CV risk. Thus, the treatment of hyperlipidemia plays a crucial role in the management of patients with CAD or those at increased risk of CAD all around the world. About 73.5 million adults in the USA have elevated LDL-cholesterol [2]. The American College of Cardiology/American Heart Association (NCEP IV) guidelines recommend prescription of evidence-based doses of statins independent of the LDL level [3].

Interestingly, most physicians prefer treating to an $\mathrm{LDL}$ goal and consider $70 \mathrm{mg} / \mathrm{dl}$ to be an appropriate target goal for people at the highest risk for cardiovascular disease [4]. However, despite achieving the target level of $70 \mathrm{mg} / \mathrm{dl}$ with high-intensity statin therapy, there is residual CV risk. Furthermore, targeting HDL and TG levels to reduce this residual risk has been proved futile [5]. Meanwhile, the recent availability of PCSK9 inhibitors has revalidated the discussion on further lowering of LDL and has brought back the age-old question: how low is in fact low enough to bring the CV risk to the minimum? 


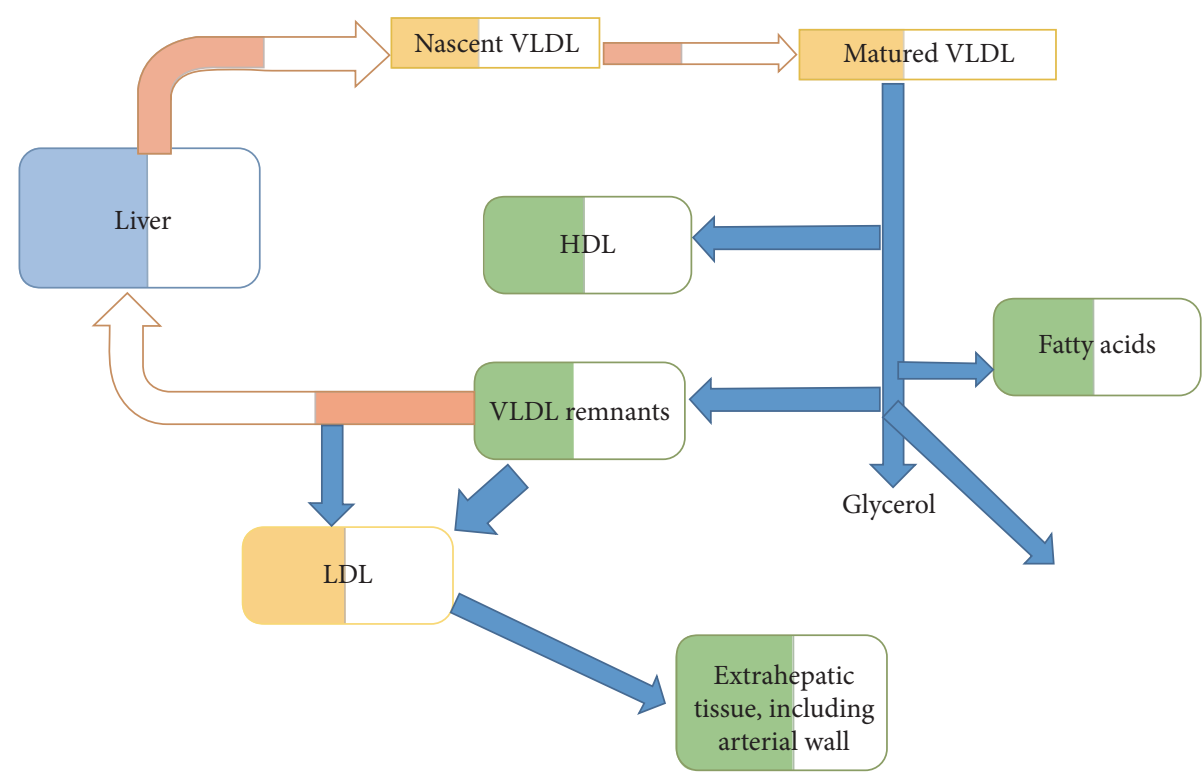

FIGURE 1: LDL metabolism.

\section{LDL Metabolism and Pathophysiology of Atherosclerosis}

The level of LDL is the single most important marker of atherosclerosis (Figure 1). Deranged LDL metabolism leads to coronary artery disease that is often fatal, especially in patients with diabetes. It has been found that not only elevated levels of LDL lead to coronary heart disease, but changes in composition can also result in the same. As we all know, cholesterol is an integral part of the plasma membrane, and a minimum level of LDL needs to be present to maintain structural integrity and sustain normal function of cells.

The development of atherosclerosis is indeed a complicated process where LDL plays a pivotal role. LDL causes endothelial damage which helps in the progression and formation of fatty streaks. Atherosclerosis, the most important factor behind the coronary vascular disease, affecting mostly medium- and large-sized arteries is characterized by the presence of modified smooth muscles, foam cells, endothelial cells, WBCs, and lipid in the center. With the growing comprehension of inflammatory process and mediators, studies have revealed that lipid-related inflammation could be cornerstone mediator for atherosclerosis [6] (Figure 2). The most likely site for plaque formation is the regions that experience low endothelial stress rather than area experiencing high stress. The plaques continue to grow into the lumen, and they experience increasingly high stress as the lumen diameter becomes narrower which ultimately contributes to the destabilization of the plaque [7]. Atherosclerosis can be prevented by implementing lifestyle modifications, controlling the risk factors of which controlling high LDL is of paramount importance.

\section{Commonly Used LDL-Lowering Drugs}

Statins. Statins inhibit the HMG-CoA reductase enzyme, the rate-controlling enzyme in the biosynthesis of cholesterol [8].
Statins lower LDL-C and triglycerides while slightly raising HDL. It is the standard of care for dyslipidemia management. Liver damage, muscle pain, and increased risk of type 2 diabetes mellitus are some of the side effects of statins, but benefits outweigh the risks [9]. They also prevent SMC migration and proliferation and impede the activation of TNF-alpha, IL-1 beta, and other interleukins which play an active role in inflammation [8].

Ezetimibe. This medication prevents the absorption of bile acid in the small intestine, lowers LDL, increases HDL slightly, and, to a little extent, lowers triglycerides. However, ezetimibe can cause myalgia and abdominal pain [10].

PCSK-9 Inhibitors. Proprotein Convertase Subtilisin/Kexin Type 9 (PCSK9) causes degradation of LDL receptors in the liver. Alirocumab and evolocumab are the two monoclonal antibodies directed against PCSK-9, and thus it prevents degradation of LDL receptors in the liver. Nasopharyngitis, reactions at the injection sites, flu-like symptoms, and muscle soreness are a few of the side effects that have been reported in the patients treated with alirocumab [11].

Fibrates, bile acid binding resins, and niacin are also used for lowering LDL-cholesterol.

\section{The Historical Perspective of Extremely Low LDL Level}

Individuals with hypobetalipoproteinemia and PCSK9 mutation have inherited natural protection from CAD. It is because of low LDL and consequently lower incidence of atherosclerosis and associated events. Patients with a total deficiency of PCSK9 have been reported to have LDL-C levels in the range of $15 \mathrm{mg} / \mathrm{dl}$ without having any adverse effects from these extremely low LDL levels [12].

Anthropological and historical evidence showed that, nearly 10,000 years ago, our ancestor hunter-gatherers, who 


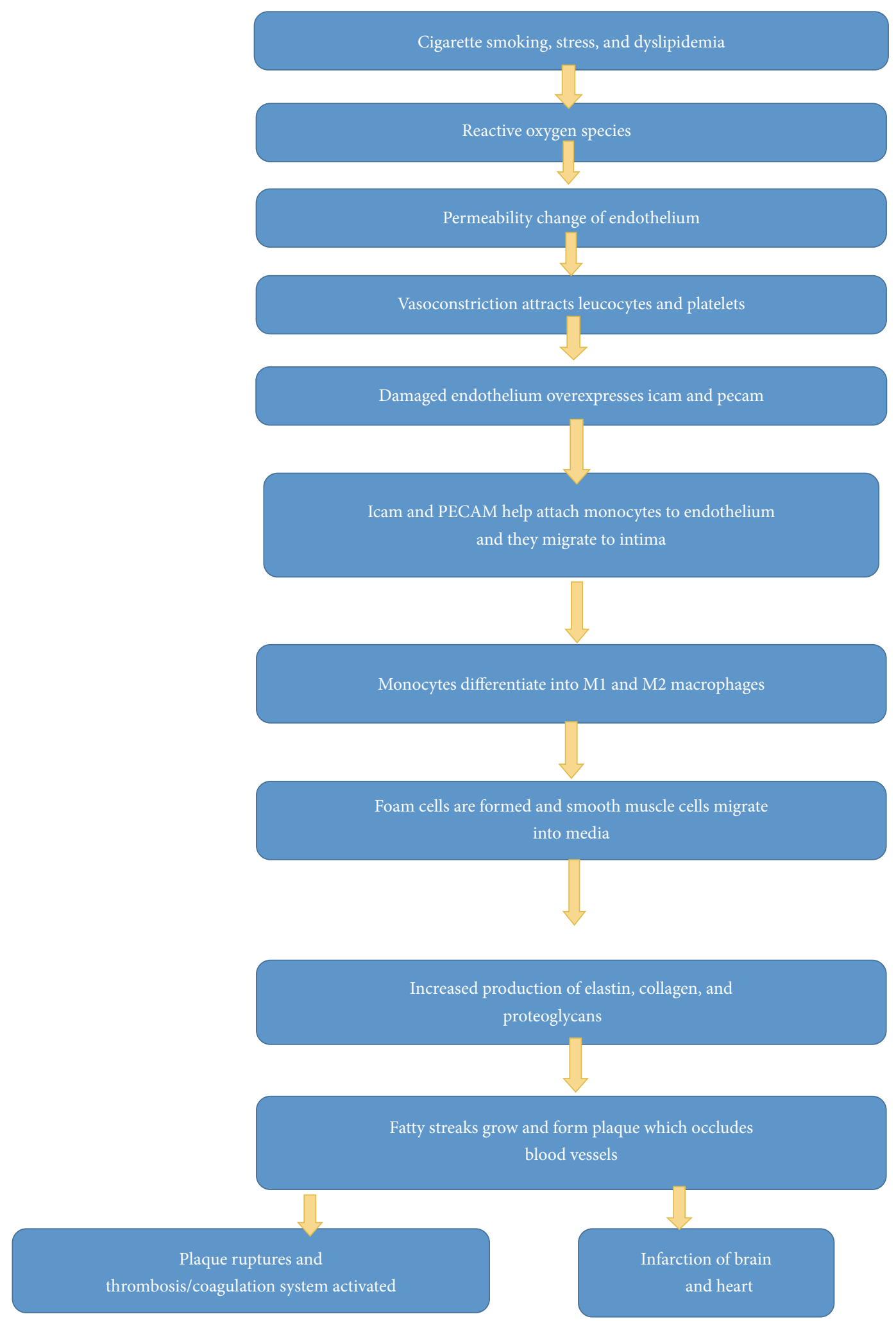

FIGURE 2: Mechanism of atherosclerosis.

were primarily dependent on wildlife diet which was mostly nuts, fruits, vegetables, and flesh of wild animals, were free from atherosclerosis with an average cholesterol level $50-75 \mathrm{mg} / \mathrm{dl}$. Only after 500 generations, after the agricultural revolution, the modern day evolved human beings are mostly reliant on processed food, refined sugars, and carbohydrates. Even the meat that we consume today is obtained from animals which are fed processed grains and corns that make 
the meat deficient in omega-3 fatty acids. In this short period, the massive changes in our dietary habits took place, which is not long enough for the genetic adaptations to happen to handle this excess load of cholesterol and this causes the rise of average serum cholesterol level to somewhat around $220-230 \mathrm{mg} / \mathrm{dl}$. These findings suggest drastic changes in our diet in comparison to genetic adaptations which are somewhat responsible for the rise of serum LDL level and increased incidence of atherosclerotic diseases [13]. Also, we know about South Asian paradox which denotes that South Asian people are more prone to develop CAD despite having within-target LDL level. So, we have to consider whether further lowering of LDL below the existing target level would help in reduction of atherosclerosis burden and CV events in those cases [14].

\section{What Defines Low and Extremely Low LDL and Its Proposed Benefits and Adverse Effects}

The LDL-C level of less than $50 \mathrm{mg} / \mathrm{dl}$ is considered low while a level of less than $20 \mathrm{mg} / \mathrm{dl}$ is considered extremely low. Intensive lipid lowering treatment has been found to halt the progression of atherosclerosis as compared to moderate lipid lowering treatment. It also regresses the atheroma plaque volume as reported by the REVERSAL (Reversal of Atherosclerosis with Aggressive Lipid Lowering) trial and ASTEROID (A Study to Evaluate the Effect of Rosuvastatin on Intravascular Ultrasound-Derived Coronary Atheroma Burden) trial. SATURN (Study of Coronary Atheroma by Intravascular Ultrasound: Effect of Rosuvastatin Versus Atorvastatin) trial also supports this [15]. An LDL level below $2.5 \mathrm{mmol} / \mathrm{l}$ can cause an atherosclerotic plaque to regress. Similarly, GLAGOV trial reported that patients who received evolocumab on a baseline treatment with statins demonstrated plaque regression in a larger number of patients as compared to placebo (64.3\% versus $47.3 \%)$ after 76 weeks of therapy [16]. In a retrospective analysis, coronary calcium score was reduced with the aggressive lowering of LDL [17].

Though the intensive lowering of LDL reduces the plaque size, there is an ongoing debate regarding its side effects. Few previous clinical trials had reported increased incidences of adverse events such as hemorrhagic strokes, dementia, depression, hematuria, and cancers with extremely low LDLC. The Dallas Heart Study $(n=12887)$, a population-based study, stretched over a period of 15 years found that a PCSK9 mutation is associated with significantly low LDL level. People with PCSK9 mutation exhibited a low incidence of CAD (a reduction of 88 percent in black and 47 percent of whites) with no increase in the hemorrhagic stroke or cancer. A person with a complete absence of PCSK9 has LDL level of about $15 \mathrm{mg} / \mathrm{dl}$, and there has not been any report of any adverse incidents [18]. The brain itself contains $25 \%$ of total cholesterol, and it is needed for maintaining its complex neuronal circuit. Blood-brain barrier is impermeable to circulatory cholesterol. This fact implies that the cholesterol regulation in the brain is not similar to that of extracerebral cholesterol. So, cholesterol level outside of the brain should not affect the brain functioning as these two cholesterol pools are different. On the surface, a target LDL level of less than $70 \mathrm{mg} / \mathrm{dl}$ may appear markedly low, but its cogency can be supported by a physiological rationale that we are born with an LDL level of $30-40 \mathrm{mg} / \mathrm{dl}$, and, at that time, the development of the brain is at its peak. The safety of low LDL level has been supported by Ray et al. They reported that the reduction of LDL levels to as low as that of a neonate is safe as well as beneficial in reducing the risk of angina, MI, or cerebrovascular disorder and total mortality [19]. These might be the levels to which humans are inherently adapted, and the levels ventured to be achieved [14]. Human brain is the most cholesterol-enriched organ but, unlike other peripheral organs, human brain is primarily dependent on de novo cholesterol synthesis rather than peripheral plasma cholesterol [20]. These above pieces of evidence lead to the hypothesis that the lowering of plasma LDL would not affect the normal brain function.

\section{Why Extremely Low or Low LDL Is Now Discussed}

Most of the statin trials showed an average of $31 \%$ of relative risk reduction which means that $69 \%$ of relative risk is still present. Despite the widespread use of statins, cardiovascular diseases and strokes are responsible for $25 \%$ of deaths worldwide. There is certainly need to address this residual risk. A meta-analysis by the Cholesterol Treatment Trialists (CTT) contributors reported that a reduction of $1 \mathrm{mmol}$ per liter in LDL-cholesterol levels results in a consistent $20 \%$ to $25 \%$ decrease in the risk of the major cardiovascular events as well as the total mortality decreasing by 12 percent [21]. PROVE IT-TIMI study noted a residual CV risk of $22.4 \%$ despite reducing LDL-C to $62 \mathrm{mg} / \mathrm{dl}$. This residual risk was targeted in various studies by modulating HDL and TG levels but showed disappointing results. However, recently PCSK9 inhibitors are emerging as a promising alternative to achieve LDL levels even below the target. Statin monotherapy upregulates the PCSK9 by $25-35 \%$ on average, along with LDL receptors in hepatocytes, which counterbalances the beneficial effects of statin. Thus, PCSK9 inhibitors would also mitigate the intrinsic counterbalancing effect of statins when given in combination [22]. However, the dilemma that continues to trouble physicians is determining how aggressively LDL needs to be treated. After the recent pooled analysis of 14 trials by Robinson et al. which showed the safety and efficacy of alirocumab in attaining low LDL level even below $15 \mathrm{mg} / \mathrm{dl}$, this topic gains momentum [23].

\section{Studies Showed Promising Results of Extremely Low LDL/Low LDL Level and Safety}

Many trials piloted to ascertain the effects of lower-thanrecommended LDL levels have reported promising results (Table 1). The TNT Trial was conducted to investigate the impact of very low LDL-C levels on major cardiovascular events compared with relatively higher LDL-C levels. The 


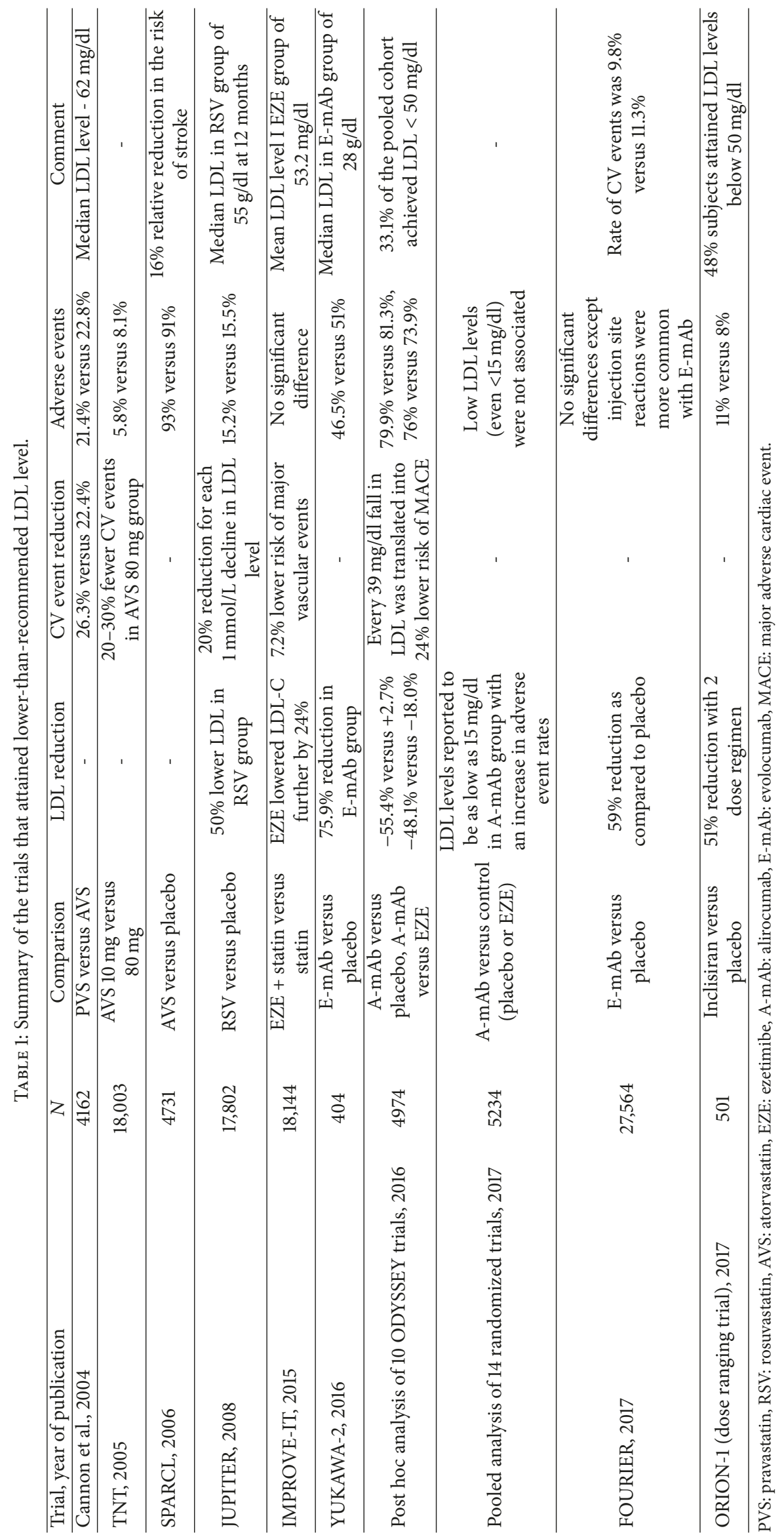


study revealed a highly significant reduction in the rate of major cardiovascular events with descending levels of LDLcholesterol $(p<0.0001)$ with a decrease of 22 percent in combined cardiovascular end point (including coronary artery disease, nonfatal MI, and resuscitated cardiac arrest and a reduction of 20 percent in cardiac deaths with lower LDL levels) [24]. Additionally, the dreaded side effects of a very low LDL-C level such as muscle pain, hemorrhagic stroke, and death due to cancer were not increased. In the IMPROVEIT (The Improved Reduction of Outcomes: Vytorin Efficacy International Trial), 18,144 participants with acute coronary syndrome were assigned to either simvastatin $(40 \mathrm{mg})$ plus ezetimibe $(10 \mathrm{mg})$ or simvastatin $(40 \mathrm{mg})$ plus placebo randomly. At seven years, the rate of combined cardiovascular death, major coronary event (nonfatal myocardial infarction, unstable angina, or coronary revascularization), or nonfatal stroke was significantly lower in the simvastatin-plusezetimibe group (32.7\% versus $34.7 \%$ ). It was observed in those who had baseline LDL level well below the current LDL goal [25]. In a study in 2007, a statin was prescribed to a group of patients with LDL-C less than $60 \mathrm{mg} / \mathrm{dl}$ who also had other comorbid conditions such as diabetes mellitus or ischemic heart disease. After a follow-up period of $2.0+/-1.4$ years, it was found that statin improved survival not only in patients taking them at the baseline level (HR, 0.58; 95\% CI, 0.38 to 0.88 ) but also in those who have LDL-C below $40 \mathrm{mg} / \mathrm{dl}$. Even patients without ischemic heart disease showed improved survival. However, there was no increased risk of elevated transaminases, malignancy, or rhabdomyolysis [26].

The JUPITER trial compared the clinical outcomes and adverse events in patients treated with rosuvastatin who attained LDL-C less than $50 \mathrm{mg} / \mathrm{dl}$ and those who did not. The study revealed reduced major cardiovascular events by $65 \%$ among those attaining LDL-C $<50 \mathrm{mg} / \mathrm{dl}$ and by $44 \%$ for the rest of the cohort. Similarly, all-cause mortality was decreased by $46 \%$ among patients achieving LDL-C $<50 \mathrm{mg} / \mathrm{dl}$ and by $20 \%$ for the remaining cohort. However, there was also a higher rate of adverse events including diabetes, hepatobiliary disorders, and insomnia in patients with LDL-C $<30 \mathrm{mg} / \mathrm{dl}$ [27]. The SPARCL (Stroke Prevention by Aggressive Reduction in Cholesterol Levels) study conducted on patients with stroke or transient ischemic attack with atorvastatin $80 \mathrm{mg}$ found that the statin reduced the chances of stroke in these groups of patients but increased the incidence of hemorrhagic stroke [28]. On the contrary, another study carried out among subjects with a history of myocardial infarction who were treated with either $80 \mathrm{mg}$ simvastatin as a part of intensive statin therapy or $20 \mathrm{mg}$ simvastatin reported no difference regarding hemorrhagic stroke after a mean follow-up period of 6.7 years (SD of 1.5 years). However, myopathy cases were reported in a higher number, among $80 \mathrm{mg}$ simvastatin users [29]. Robinson et al. evaluated the safety of alirocumab. They described LDL-C levels to be as low as $15 \mathrm{mg} / \mathrm{dl}$ and did not report any adverse neurocognitive event, although a nonsignificant increase in cataract incidence seemed to be more in the group achieving LDL-C levels $<25 \mathrm{mg} / \mathrm{dl}$ [23]. In the same vein, Sabatine et al. did not report any significant increase in adverse reactions with very low LDL-C. Also, Prostate Cancer Prevention Trial indicated that low cholesterol is associated with reduced risk of high grade of prostate cancer [30]. A Retrospective Observational Study conducted in Quebec, Canada, on patients admitted with acute myocardial infarction concluded that high dose statin use might be associated with significant reduction in the cancer incidence [31]. The substudy of PROVE IT-TIMI 22 investigating $80 \mathrm{mg}$ atorvastatin versus $40 \mathrm{mg}$ pravastatin also proved that achieving LDL-C level below the expected level ( 80 to $100 \mathrm{mg} / \mathrm{dl}$ ) is not associated with increased adverse events [32].

A meta-analysis by Boekholdt et al. reported an increased risk of hemorrhagic stroke in those with very low levels of LDL as compared to moderately low levels. However, the absolute number was low, and the statistical power was therefore insufficient to draw a definite conclusion. Furthermore, they believed that significantly lower risk of cerebrovascular events outweighed the potential for hemorrhagic stroke [33]. A Phase 3 Study of Evolocumab showed no increase in the adverse events despite a median LDL level of $26-36 \mathrm{mg} / \mathrm{dl}$ over 12 weeks [34]. The most recent data about safety and efficacy of low LDL comes from the FOURIER trial. They have shown a significant reduction of LDL from a baseline value of $92 \mathrm{mg} / \mathrm{dl}$ to $30 \mathrm{mg} / \mathrm{dl}$ with evolocumab. Most importantly, there was a significant decrease in the risk of major cardiovascular events without any major rise in the adverse events. They reported a $17 \%$ decrease in the cardiovascular death, myocardial infarction, and stroke on lowering the LDL to $43 \mathrm{mg} / \mathrm{dl}$ while reducing the LDL levels further to $22 \mathrm{mg} / \mathrm{dl}$ decreased the risk to $20 \%$. Additionally, they reported consistent clinical improvements per unit reduction in LDL [35]. A post hoc analysis of 10 ODYSSEY trials comparing alirocumab with the control indicated that low LDL-C was associated with a lower incidence of major adverse cardiovascular events with no significant increment in the treatmentemergent adverse reactions [19].

Very recently, a prespecified safety analysis of IMPROVEIT involving 15281 patients showed that patients $(n=971)$ with LDL level below $30 \mathrm{mg} / \mathrm{dl}$ had no increased adverse events over six years' follow-up [36].

\section{Future Directions and Ongoing Studies}

Low and extremely low LDL-C levels are being supported widely; however, many have raised concerns about their long-term effects which still stands unexplored. The mystery behind the advantages and disadvantages of prolonged exposure to pharmacologically induced low LDL levels needs to be unveiled.

A common finding among the LDL-lowering trials was the time lag between the onset of LDL lowering and the appearance of full clinical benefits regarding risk reduction presenting itself as another lacuna in the better understanding of the link between LDL-C lowering and CV risk reduction.

Bringing LDL to very low levels with statin monotherapy poses safety concerns in some patients. While the emergence of PCSK9 inhibitors has appeared to solve the problem, the cost effectiveness of treatment with PCSK9 inhibitors remains questionable. The immunogenic effects of the PCSK9 inhibitors varying from mild injection site reactions to 
anaphylaxis and loss of drug efficacy need to be scrutinized further. Recently SPIRE trial showed antibodies against the murine component of bococizumab in $15-20 \%$ of patients and a neutralizing antidrug antibody was seen only in $1.3 \%$ patients on alirocumab [37]. Poor adherence to the treatment due to multiple injections is another issue with the PCSK9 monoclonal antibodies.

A therapeutic strategy involving small (21-25 bp) interfering RNA (siRNA) targeting PCSK9 has gained our attraction recent past. Inclisiran, a novel therapeutic drug that inhibits PCSK9 through RNA interference, has shown encouraging results in an average reduction of LDL by $51 \%$ with only a 2 dose regime over a period of 9 months. This was investigated in a clinical phase 2 trial, ORION-1. The result of this trial is encouraging as ease of using this drug will be impactful as it needs only one or two injections over six-month to 1year period [38]. Nevertheless, the impact on cardiovascular outcomes is yet to be studied in ORION-4. None of the studies have thus far mentioned the duration of treatment with PCSK9 inhibitors needed to maintain the risk reduction.

Peptide-based anti-PCSK9 vaccines are being tested on mice which supposedly have the potential to control LDL level for a longer duration [39].

Several ongoing studies are aiming to enhance our knowledge regarding the safety of low LDL and reduction in cardiovascular risk. In ODYSSEY OUTCOMES, a placebo-controlled phase 3 trial, 18600 post-MI patients are being randomized to alirocumab or placebo arm. It intends to compare the effects of alirocumab with placebo on the occurrence of cardiovascular events over a period of 64 months. The ODYSSEY APPRISE is a multicountry, multicenter phase 3 study aimed at investigating the safety of alirocumab in patients with severe hypercholesterolemia over a period of 30 months. TAUSSIG is another ongoing study designed to assess the long-term safety, tolerability, and efficacy of evolocumab in patients with severe hypercholesterolemia. Meanwhile, PACMAN-AMI is evaluating the effects of PCSK9 inhibition on the morphology of coronary plaque in patients with acute myocardial infarction.

\section{Conclusion}

In summary, the residual risk despite achieving the current target LDL levels needs to be addressed. Although the clinical benefits of lowering LDL have been well stated, their longterm consequences are still under investigation. Many trials conducted in the past were successful in reducing the LDL levels well below the target with a consequent reduction in $\mathrm{CV}$ risk. Though there is ample evidence that low LDL does protect from residual CV risk, there have also been a few studies claiming an increasing number of adverse events with low and extremely low LDL levels. Nevertheless, we have to wait for the result of ongoing trials to have a conclusive answer on the long-term effect of lowering the current LDL goal.

\section{Conflicts of Interest}

The authors declare that there are no conflicts of interest.

\section{References}

[1] D. Mozaffarian, E. J. Benjamin, A. S. Go et al., "Heart disease and stroke statistics-2016 update," in Circulation, vol. 132, American Heart Association, 2015.

[2] D. Mozaffarian, E. J. Benjamin, A. S. Go et al., "Heart Disease and Stroke Statistics-2015 Update," in Circulation, vol. 131, American Heart Association, 2014.

[3] N. J. Stone, J. Robinson, and A. H. Lichtenstein, "2013 ACC/ AHA guideline on the treatment of blood cholesterol to reduce atherosclerotic cardiovascular risk in adults: a report of the American College of Cardiology/American Heart Association Task Force on Practice Guidelines," Circulation, 2013.

[4] M. Nayor and R. S. Vasan, "Recent update to the us cholesterol treatment guidelines: A comparison with international guidelines," Circulation, vol. 133, no. 18, pp. 1795-1806, 2016.

[5] W. E. Boden, J. L. Probstfield, T. Anderson et al., "Niacin in patients with low HDL cholesterol levels receiving intensive statin therapy," The New England Journal of Medicine, vol. 365, no. 24 , pp. 2255-2267, 2011.

[6] C. Weber and H. Noels, "Atherosclerosis: current pathogenesis and therapeutic options," Nature Medicine, vol. 17, no. 11, pp. 1410-1422, 2011.

[7] J. J. Wentzel, Y. S. Chatzizisis, F. J. H. Gijsen, G. D. Giannoglou, C. L. Feldman, and P. H. Stone, "Endothelial shear stress in the evolution of coronary atherosclerotic plaque and vascular remodelling: Current understanding and remaining questions," Cardiovascular Research, vol. 96, no. 2, pp. 234-243, 2012.

[8] K. Pahan, "Lipid-lowering drugs," Cellular and Molecular Life Sciences, vol. 63, no. 10, pp. 1165-1178, 2006.

[9] WebMD, "Which Medicines Lower "Bad" (LDL) Cholesterol," http://www.webmd.com/cholesterol-management/guide/cholesterol-lowering-medication\#1, 2017.

[10] Cholesterol Medications: Consider The Options, Mayo Clinic, 2015, http://www.mayoclinic.org/diseases-conditions/high-bloodcholesterol/in-depth/cholesterol-medications/art-20050958.

[11] https://www.mayoclinic.org/drugs-supplements/alirocumabsubcutaneous-route/side-effects/drg-20151256.

[12] J. D. Horton, J. C. Cohen, and H. H. Hobbs, "PCSK9: a convertase that coordinates LDL catabolism," Journal of Lipid Research, vol. 50, pp. S172-S177, 2009.

[13] J. H. O’Keefe Jr. and L. Cordain, “Cardiovascular Disease Resulting from a Diet and Lifestyle at Odds with Our Paleolithic Genome: How to Become a 21st-Century Hunter-Gatherer," Mayo Clinic Proceedings, vol. 79, no. 1, pp. 101-108, 2004.

[14] J. H. O’Keefe Jr., L. Cordain, W. H. Harris, R. M. Moe, and R. Vogel, "Optimal low-density lipoprotein is 50 to $70 \mathrm{mg} / \mathrm{dl}$ : Lower is better and physiologically normal," Journal of the American College of Cardiology, vol. 43, no. 11, pp. 2142-2146, 2004.

[15] T. Dave, J. Ezhilan, H. Vasnawala, and V. Somani, "Plaque regression and plaque stabilisation in cardiovascular diseases," Indian Journal of Endocrinology and Metabolism, vol. 17, no. 6, p. 983, 2013.

[16] S. J. Nicholls, R. Puri, T. Anderson et al., "Effect of evolocumab on progression of coronary disease in statin-treated patients: The GLAGOV randomized clinical trial," Journal of the American Medical Association, vol. 316, no. 22, pp. 2373-2384, 2016.

[17] B. Ibanez, G. Vilahur, and J. J. Badimon, "Plaque progression and regression in atherothrombosis," Journal of Thrombosis and Haemostasis, vol. 5, no. 1, pp. 292-299, 2007. 
[18] T. McCormack, R. Dent, and M. Blagden, "Very low LDLC levels may safely provide additional clinical cardiovascular benefit: the evidence to date," International Journal of Clinical Practice, vol. 70, no. 11, pp. 886-897, 2016.

[19] K. K. Ray, H. N. Ginsberg, M. H. Davidson et al., "Reductions in Atherogenic Lipids and Major Cardiovascular Events: A Pooled Analysis of 10 ODYSSEY Trials Comparing Alirocumab with Control," Circulation, vol. 134, no. 24, pp. 1931-1943, 2016.

[20] M. Orth and S. Bellosta, "Cholesterol: its regulation and role in central nervous system disorders," Cholesterol, vol. 2012, Article ID 292598, 19 pages, 2012.

[21] C. Baigent, A. Keech, and P. M. Kearney, "Efficacy and safety of cholesterol-lowering treatment: prospective meta-analysis of data from 90,056 participants in 14 randomised trials of statins," The Lancet, vol. 366, no. 9493, pp. 1267-1278, 2005.

[22] N. E. Lepor and D. J. Kereiakes, “The PCSK9 inhibitors: A novel therapeutic target enters clinical practice," American Health and Drug Benefits, vol. 8, no. 9, pp. 483-488, 2015.

[23] J. G. Robinson, R. S. Rosenson, M. Farnier et al., "Safety of Very Low Low-Density Lipoprotein Cholesterol Levels With Alirocumab: Pooled Data From Randomized Trials," Journal of the American College of Cardiology, vol. 69, no. 5, pp. 471-482, 2017.

[24] J. C. LaRosa, S. M. Grundy, J. J. P. Kastelein et al., "Safety and efficacy of Atorvastatin-induced very low-density lipoprotein cholesterol levels in patients with coronary heart disease (a post hoc analysis of the treating to new targets [TNT] study)," American Journal of Cardiology, vol. 100, no. 5, pp. 747-752, 2007.

[25] S. A. Murphy, C. P. Cannon, M. A. Blazing et al., "Reduction in Total Cardiovascular Events with Ezetimibe/Simvastatin PostAcute Coronary Syndrome the IMPROVE-IT Trial," Journal of the American College of Cardiology, vol. 67, no. 4, pp. 353-361, 2016.

[26] N. J. Leeper, R. Ardehali, E. M. DeGoma, and P. A. Heidenreich, "Statin use in patients with extremely low low-density lipoprotein levels is associated with improved survival," Circulation, vol. 116, no. 6, pp. 613-618, 2007.

[27] J. Hsia, J. G. MacFadyen, J. Monyak, and P. M. Ridker, "Cardiovascular event reduction and adverse events among subjects attaining low-density lipoprotein cholesterol $<50 \mathrm{mg} / \mathrm{dl}$ with rosuvastatin: The JUPITER trial (justification for the use of statins in prevention: An intervention trial evaluating rosuvastatin)," Journal of the American College of Cardiology, vol. 57, no. 16, pp. 1666-1675, 2011.

[28] L. B. Goldstein, P. Amarenco, M. Szarek et al., "Hemorrhagic stroke in the Stroke Prevention by Aggressive Reduction in Cholesterol Levels study.," Neurology, vol. 70, no. 24, pp. 23642370, 2008.

[29] J. Armitage, L. Bowman, K. Wallendszus et al., "Intensive lowering of LDL cholesterol with $80 \mathrm{mg}$ versus $20 \mathrm{mg}$ simvastatin daily in 12,064 survivors of myocardial infarction: a doubleblind randomised trial," Lancet, vol. 376, no. 9753, pp. 16581669, 2010.

[30] E. A. Platz, C. Till, P. J. Goodman et al., "Men with low serum cholesterol have a lower risk of high-grade prostate cancer in the placebo arm of the prostate cancer prevention trial," Cancer Epidemiology, Biomarkers \& Prevention, vol. 18, no. 11, pp. 28072813, 2009.

[31] I. Karp, H. Behlouli, J. LeLorier, and L. Pilote, "Statins and Cancer Risk," American Journal of Medicine, vol. 121, no. 4, pp. 302-309, 2008.
[32] S. D. Wiviott, C. P. Cannon, D. A. Morrow, K. K. Ray, M. A. Pfeffer, and E. Braunwald, "Can low-density lipoprotein be too low? The safety and efficacy of achieving very low low-density lipoprotein with intensive statin therapy: A PROVE IT-TIMI 22 substudy," Journal of the American College of Cardiology, vol. 46, no. 8, pp. 1411-1416, 2005.

[33] S. M. Boekholdt, G. K. Hovingh, S. Mora et al., "Very low levels of atherogenic lipoproteins and the risk for cardiovascular events: a meta-analysis of statin trials," Journal of the American College of Cardiology, vol. 64, pp. 485-494, 2014.

[34] A. Kiyosue, N. Honarpour, C. Kurtz, A. Xue, S. M. Wasserman, and A. Hirayama, "A Phase 3 Study of Evolocumab (AMG 145) in Statin-Treated Japanese Patients at High Cardiovascular Risk," American Journal of Cardiology, vol. 117, no. 1, pp. 40-47, 2016.

[35] M. S. Sabatine, R. P. Giugliano, A. C. Keech et al., "Evolocumab and clinical outcomes in patients with cardiovascular disease," The New England Journal of Medicine, vol. 376, no. 18, pp. 17131722, 2017.

[36] R. P. Giugliano, S. D. Wiviott, M. A. Blazing et al., "Longterm safety and efficacy of achieving very low levels of lowdensity lipoprotein cholesterol: A prespecified analysis of the IMPROVE-IT trial," JAMA Cardiology, vol. 2, no. 5, pp. 547555, 2017.

[37] P. M. Ridker, J. Revkin, P. Amarenco et al., "Cardiovascular efficacy and safety of bococizumab in high-risk patients," The New England Journal of Medicine, vol. 376, no. 16, pp. 1527-1539, 2017.

[38] K. K. Ray, U. Landmesser, L. A. Leiter et al., "Inclisiran in patients at high cardiovascular risk with elevated LDL cholesterol," The New England Journal of Medicine, vol. 376, no. 15, pp. 1430-1440, 2017.

[39] G. Galabova, S. Brunner, G. Winsauer et al., "Peptide-based anti-PCSK9 vaccines-an approach for long-term LDLc management," PLoS ONE, vol. 9, no. 12, Article ID el14469, 2014. 


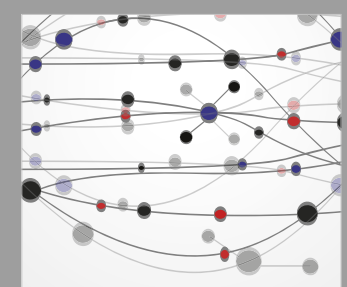

The Scientific World Journal
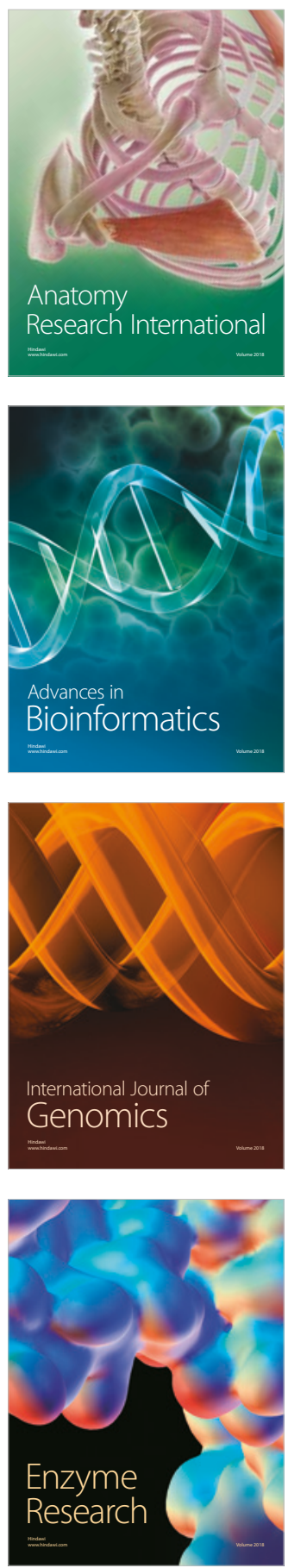
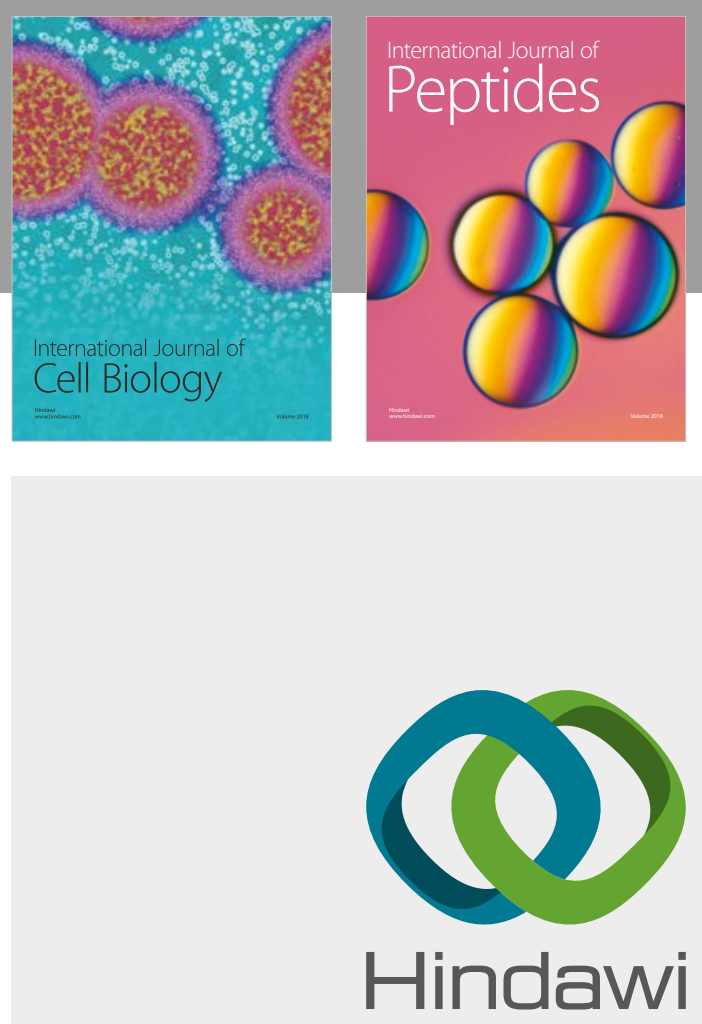

Submit your manuscripts at

www.hindawi.com
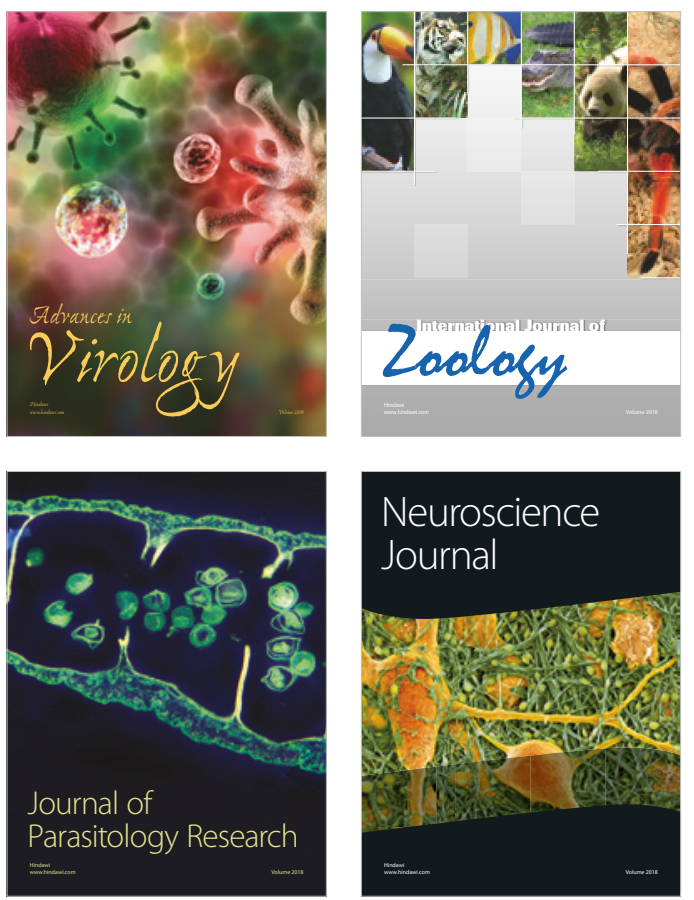
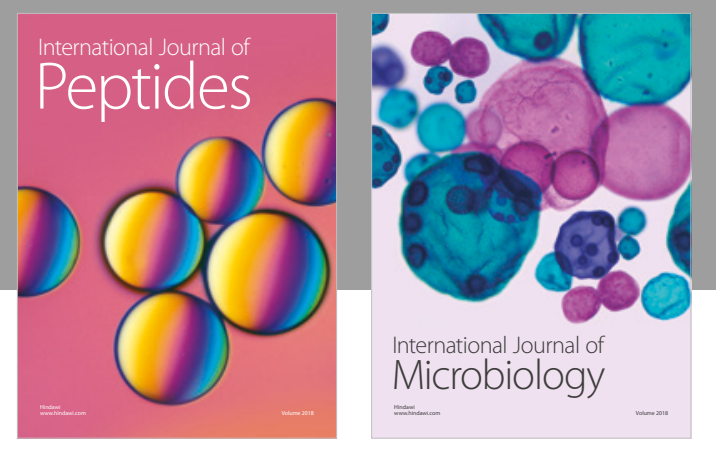

nternational Journal of Microbiology
Journal of
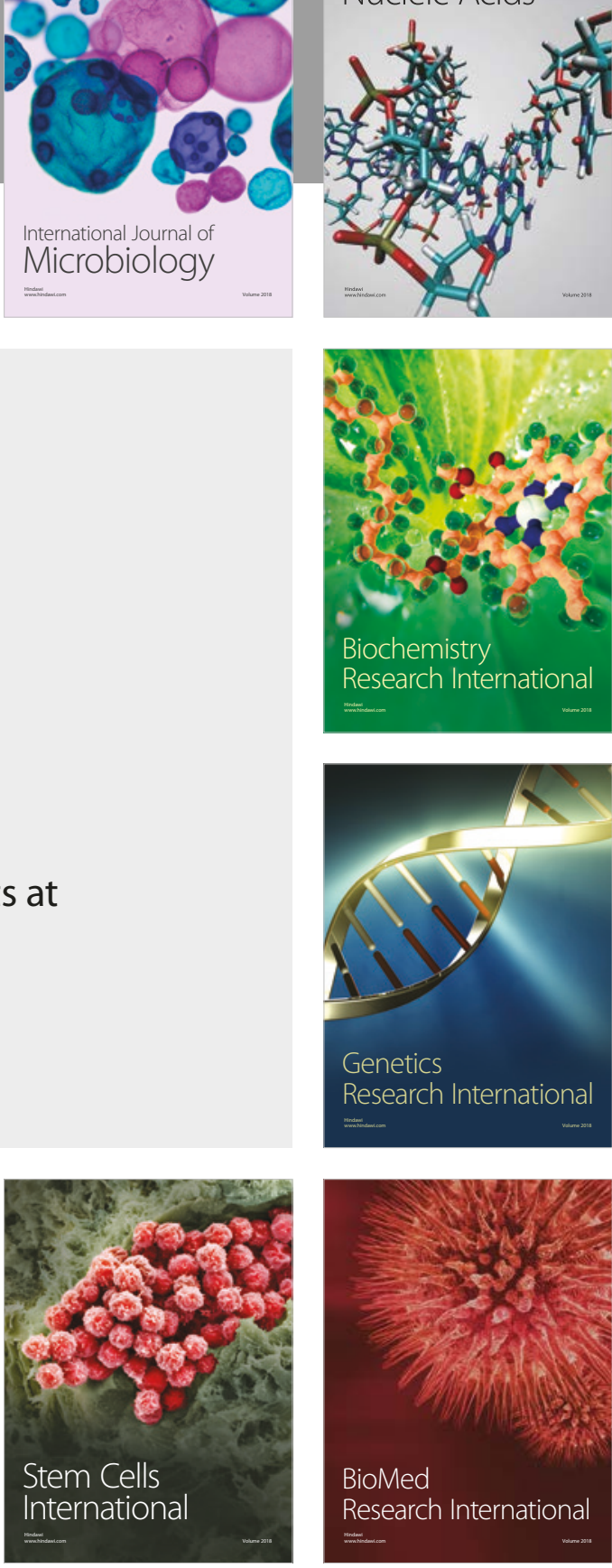
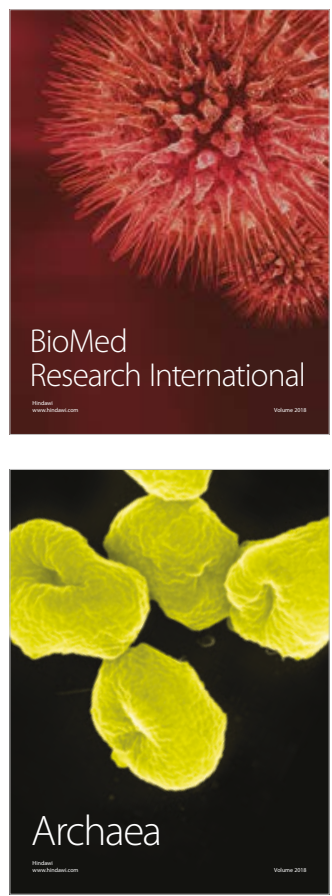